Zur Ausgestaltung handelbarer Flächenausweisungsrechte

\section{Innovative Instrumente zur Steuerung des Flächenverbrauchs}

Die hohe Flächeninanspruchnahme von gegenwärtig etwa 110 Hektar pro Tag gehört nach wie vor zu den ungelösten Umweltproblemen in Deutschland. Doch der anhaltende Flächenverbrauch kann durch eine Kombination handelbarer Flächenausweisungsrechte und planerischer Instrumente ökonomisch effizient vermindert werden. Von Peter Michaelis

D ie hohe Rate der siedlungs- und verkehrsbedingten Flächeninanspruchnahme von gegenwärtig etwa 110 Hektar pro Tag gehört nach wie vor zu den ungelösten Umweltproblemen in Deutschland. Ihre Auswirkungen beeinträchtigen nicht nur wichtige Funktionen des Naturhaushalts sondern auch die Lebensqualität der Menschen.

Die hohe Rate der siedlungs- und verkehrsbedingten Flächeninanspruchnahme von gegenwärtig etwa 110 Hektar pro Tag gehört nach wie vor zu den ungelösten Umweltproblemen in Deutschland. Ihre Auswirkungen beeinträchtigen nicht nur wichtige Funktionen des Naturhaushalts sondern auch die Lebensqualität der Menschen. Im Rahmen des existierenden deutschen Planungssystems konnte bisher allein durch planerische Mittel keine ausreichende Steuerungswirkung zur Reduzierung der Flächeninanspruchnahme erzielt werden.

Die Flächeninanspruchnahme ist aufgrund wirtschaftlicher Stagnation in den vergangenen Jahren etwas zurückgegangen und demografische Faktoren könnten weiterhin dazu beitragen, dass weniger Flächen bebaut werden. Trotzdem ist das von der Bundesregierung in ihrer Nachhaltigkeitsstrategie formulierte Ziel, eine Reduzierung der Flächeninanspruchnahme auf 30 Hektar pro Tag zu erreichen, mit den gegenwärtigen Mitteln der räumlichen Planung nicht zu erreichen.

Eine ausschließliche Steuerung der Flächeninanspruchnahme durch die räumliche Planung hätte zudem eine star- ke Einschränkung der Abwägungsmöglichkeiten der Kommunen zur Folge und würde aus ökonomischer Sicht keine ausreichend flexible und bedarfsgerechte Allokation im Raum gewährleisten.

\section{Instrumentenmix statt Flächensteuer}

Einzelne ökonomische Instrumente, wie Abgaben und Steuern oder eine ökologisch orientierte Erweiterung des kommunalen Finanzausgleichs, sind nicht in der Lage, eine hinreichend starke Senkung der Flächeninanspruchnahme zu bewirken (SRU 2002):

- Um eine ausreichende quantitative Wirkung zu entfalten, müssten die entsprechenden Abgaben und Steuern eine Höhe erreichen, die mit unerwünschten Auswirkungen auf die wirtschaftliche Entwicklung verbunden wäre und jenseits der politisch umsetzbaren Grenze liegen dürfte.

- Zuweisungen aus einem ökologischen Finanzausgleich müssten, wenn sie nicht nur in Räumen mit geringer Siedlungsdynamik Wirkung entfalten sollten, ebenfalls vergleichsweise hoch ausfallen, wodurch sich erhebliche Finanzierungsprobleme ergäben.

Vor diesem Hintergrund hat der Sachverständigenrat für Umweltfragen in seinem Hauptgutachten 2004 zur wirksamen Reduzierung der Flächeninanspruchnahme einen Instrumentenmix empfohlen, der auf einer Kombination ökonomischer und planerischer Instrumente beruht (SRU 2004; v. Haaren 2005). Im Zentrum steht dabei ein System handelbarer Flächenausweisungsrechte, die den Kommunen zugewiesen werden. Diese Rechte begrenzen die maximal ausweisbare Fläche durch hoheitliche Zielfestlegung auf den übergeordneten Ebenen von Bund und Ländern für einen bestimmten Zeitraum. Nicht benötigte Ausweisungsrechte können von den Kommunen an einer Börse verkauft und zusätzliche Rechte dort erworben werden. Dadurch wird eine unter ökonomischen Gesichtspunkten effiziente Verteilung der Flächeninanspruchnahme im Raum bewirkt.

Zugleich werden Innovationspotenziale zur Verwirklichung flächensparender Nutzungen aktiviert. Für die Erstzuteilung der Rechte bestehen unterschiedliche Optionen. Grundsätzlich empfiehlt sich eine kostenlose Zuteilung, da hierdurch - im Gegensatz zu einer Versteigerung - eine zusätzliche finanzielle Belastung der Kommunen vermieden und die politische Akzeptanz des Systems erhöht wird. Der Zuteilung können verschiedene Kriterien zugrunde gelegt werden, die jeweils unterschiedliche Steuerungsziele repräsentieren. Welches Kriterium Verwendung finden soll, ist letztendlich eine politisch-normative Entscheidung.

\section{Flächenhandel effizient gestalten}

Beispielweise würde eine Zuteilung nach der Einwohnerzahl die Flächeninanspruchnahme in stark verdichteten Gebieten fördern. Damit würde honoriert, dass die Kommunen in Abhängigkeit von ihrer Einwohnerzahl Infrastrukturen bereitstellen müssen. Eine Zuteilung nach der jeweiligen Fläche der Kommune würde flächenreiche und häufig strukturschwache Räume bevorzugen. Zieht man die Siedlungs- und Verkehrsfläche als Basis heran, so werden bereits stark entwickelte und gut erschlossene Räume unterstützt. Wird hingegen die Vergangenheitsentwicklung zugrunde gelegt, so werden Gebiete mit hoher Dynamik gefördert. Dabei ist die ökonomische Effizienz des Flächenhandels unabhängig davon, welcher Zuteilungsschlüssel gewährt wird. Sie wäre 
allerdings umso höher, je größer der Raum wäre, in dem die Flächenausweisungsrechte gehandelt werden könnte. Idealerweise würde das System also auf Bundesebene eingeführt werden.

\section{Integration von Naturschutzaspekten}

Eine reine Steuerung über Flächenausweisungsrechte hätte jedoch den Nachteil, dass raumkonkrete Umweltund Naturschutzaspekte bei der Verteilung der Flächeninanspruchnahme im Raum nicht berücksichtigt werden. Deshalb bedarf der Flächenhandel einer Flankierung durch die Raumplanung, die ihn gleichsam zähmen muss. In Kombination mit der Landschaftsplanung ist sie das geeignete Instrument für eine Berücksichtigung von raumkonkreten Umwelt- und Naturschutzaspekten. In der Frage nach der sinnvollen Kombination von Flächenhandel und Raumplanung liegt eine der entscheidenden konzeptionellen Herausforderungen.

Für eine Einflussnahme der Raumplanung im Zusammenspiel mit dem Flächenhandel gibt es zwei Ansatzpunkte. Zum einen wäre es möglich, dass sich die Erstzuteilung der Flächenausweisungsrechte an raumordnerischen Kriterien orientiert. Diese Option wird häufig in Betracht gezogen, ist jedoch nicht $\mathrm{zu}$ empfehlen. Denn Gemeinden mit einem hohen Anteil umweltempfindlicher oder für den Natur- und Umweltschutz bedeutsamer Flächen würden im Rahmen dieser Variante nur eine geringere Anzahl von Flächenausweisungsrechten erhalten. Diese Gemeinden würden damit von Anfang an benachteiligt und dennoch nicht einmal daran gehindert, wertvolle Flächen in Anspruch zu nehmen. Zum anderen, und dies ist die vorzuziehende Option, könnte die Raumplanung auf Grundlage der Landschaftsplanung Flächen vorgeben, auf denen eine Siedlungsentwicklung nicht oder nur unter Einschränkungen stattfinden darf, sogenannte ökologische Tabuflächen.

Der damit abgesteckte Rahmen könnte anschließend durch qualitative Festlegungen der Nutzungen nach den Vorga- ben der Raumplanung sowie des sonstigen Planungs- und Umweltrechts auch auf kommunaler Ebene ausgefüllt werden.

Nur diese Option garantiert, dass die Nutzungsansprüche auf die unter umweltund raumplanerischen Gesichtspunkten geeigneten Flächen konzentriert werden.

\section{Eine Gesamtbilanz der verfügbaren Flächen}

Um zu verhindern, dass die ökonomische Effizienz des Flächenhandels durch eine übermäßige Planungssteuerung vollständig verloren geht, und um sicherzustellen, dass ein Spielraum für den Flächenhandel verbleibt, sollte eine Gesamtbilanz der für die Siedlungsentwicklung verfügbaren beziehungsweise davon ausgenommenen Gebiete auf Landes- oder Bundesebene geführt sowie einheitliche Kriterien für die Ausweisung der Tabuflächen vorgegeben werden. Im Rahmen dieser Vorgehensweise würden die Vorteile handelbarer Flächenausweisungsrechte mit denen der Planungssteuerung vereint und die Effizienz des Flächenhandels nur insoweit beeinträchtigt, wie die Möglichkeiten zur Flächeninanspruchnahme durch die Ausweisung von Tabuflächen vermindert würden.

\section{Literatur}

SRU (Sachverständigenrat von Umweltfragen): Sondergutachten Naturschutz. Baden-Baden 2002.

SRU (Sachverständigenrat von Umweltfragen): Umweltgutachten 2004. Baden-Baden 2004. v. Haaren, Ch. / Michaelis, P.: Handelbare Flächenausweisungsrechte und Planung. Informationen zur Raumentwicklung, 4/5 2005 S. 12-29.

\section{AUTOR + KONTAKT}

Dr. Peter Michaelis ist Inhaber des Lehrstuhls für Umwelt- und Ressourcenökonomik an der wirtschaftswissenschaftlichen Fakultät der Universität Augsburg und seit August 2000 Mitglied im Sachverständigenrat für Umweltfragen, Berlin. Wirtschaftswissenschaftliche Fakultät der Universität Augsburg, Universitätsstraße 16, 8615 Augsburg. Tel.: 0821/598-4057, Fax: 0821/598-4217, E-Mail: peter.michaelis@wiwi.uni-augsburg.de politische ökologie

Die Zeitschrift für Querdenker und Vordenkerinnen

Grünbuch Europa

Von nachhaltigen Visionen und umweltpolitischen Realitäten

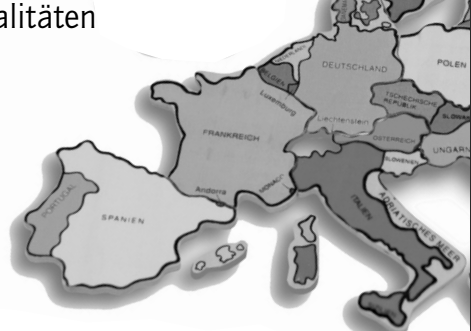

Wir sind EU-Ratspräsident! Sechs Monate hat Deutschland Zeit, um den angeschlagenen Ruf als umweltpolitischer Vorreiter aufzupolieren. Die politische ökologie ${ }^{102-103}$ bringt Sie bei allen wichtigen Debatten auf den neuesten Stand - von der EU-Nachhaltigkeitsstrategie über die Klima- und Energiepolitik bis zum Biodiversitätsziel 2010.

_Wie fährt Europas Verkehr aus der umwelt politischen Sackgasse?

_Wer knackt die Brüsseler Subventionslogik in der Landwirtschaft?

_Wie funktioniert Lobbying in Brüssel?

Mit Beiträgen von C. Hey, A. Baumann, J. Porritt, F. Wolff, F. Schwalba-Hoth, H. Lorenzen, C. Knill, F. Schmidt u.v.m.

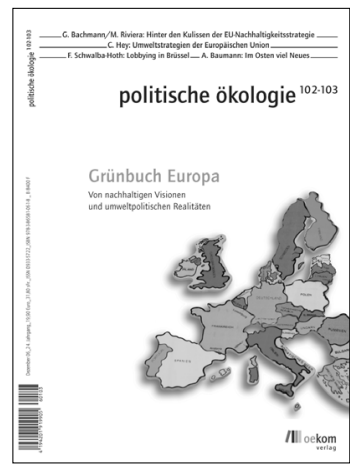

Stillen Sie Ihre Neugier!

$$
\begin{aligned}
& \text { _Fordern Sie Heft 102-103 an! } \\
& \text { _19,90 EUR (inkl. Versand)/31,80 CHF } \\
& \text { _ISBN 978-3-86581-061-8 } \\
& \text { _oekom verlag } \\
& \text { _Fax +49/(0)89/54 41 84-49 } \\
& \text { _www.oekom.de }
\end{aligned}
$$

neugier@oekom.de 
(c) 20I0 Authors; licensee IÖW and oekom verlag. This is an article distributed under the terms of the Creative Commons Attribution Non-Commercial No Derivates License (http://creativecommons.org/licenses/by-nc-nd/3.o/), which permits unrestricted use, distribution, and reproduction in any medium, provided the original work is properly cited. 